\title{
Finding our way through the labyrinth of dementia biomarkers
}

\author{
Gaël Chételat ${ }^{1}$ - Javier Arbizu ${ }^{2}$ - Henryk Barthel ${ }^{3}$. Valentina Garibotto ${ }^{4}$. Adriaan A. Lammertsma ${ }^{5,6} \cdot \operatorname{lan}_{\text {Law }^{7}}$. \\ Silvia Morbelli ${ }^{8} \cdot$ Elsmarieke van de Giessen $^{9} \cdot$ Alexander Drzezga $^{10,11,12}$
}

Published online: 20 April 2021

(C) The Author(s), under exclusive licence to Springer-Verlag GmbH Germany, part of Springer Nature 2021

In 1984, consensual criteria for Alzheimer's disease (AD) were published, which were fully based on symptoms as tracked by clinical and neuropsychological examinations [1]. More than 20 years later, revised criteria have been proposed, progressively integrating biomarkers, until 2018 when the newly proposed research criteria exclusively relied on biomarkers [2-4]. This shift from one extreme to the other has been controversial [5]. Wherever we stand in this debate, it must be acknowledged that early diagnosis of neurodegeneration as well as reliable etiological diagnosis of the causes underlying cognitive decline remains challenging on the basis of merely clinical assessment. Biomarkers have demonstrated great value in that respect and, accordingly, they are playing an increasing role. There is also general agreement that combining biomarkers improves diagnostic accuracy $[6,7]$. Consequently, biomarker development is booming, broadening the spectrum of available modalities (magnetic resonance imaging (MRI), positon emission tomography (PET), cerebrospinal fluid (CSF), upcoming blood biomarkers), and the pathologies to be assessed (neurodegeneration, amyloid, tau $\alpha$ -

This article is part of the Topical Collection on Neurology

Gaël Chételat

chetelat@cyceron.fr

1 Normandie Univ, UNICAEN, INSERM, U1237, PhIND

"Physiopathology and Imaging of Neurological Disorders", Institut Blood and Brain@ Caen-Normandie, Cyceron, 14000 Caen, France

2 Department of Nuclear Medicine, University of Navarra Clinic, Pamplona, Spain

3 Department of Nuclear Medicine, University Hospital of Leipzig, Leipzig, Germany

4 Division of Nuclear Medicine and Molecular Imaging, Geneva University Hospitals and NIMTlab, Geneva University, Geneva, Switzerland

5 Department of Radiology and Nuclear Medicine, Amsterdam University Medical Centers, Vrije Universiteit, Amsterdam, The Netherlands synuclein, neuroinflammation, synaptic density, etc.) (Fig. 1). However, faced with this arsenal of biomarkers, uncertainty has arisen regarding appropriate combination and/or order in which these biomarkers should be used in diagnostic evaluations.

In an article published as a Personal View in the Lancet Neurology in November 2020 [8], we thus proposed a diagnostic algorithm aiming at providing consensus recommendations for the optimal ordering of the most validated biomarkers into meaningful sequences depending on the clinical presentation. This article was the end result of intensive debates and discussions from a multidisciplinary group of experts and opinion leaders from all over the world, comprised of nuclear medicine physicians, radiologists, neurologists, geriatricians, psychiatrists, clinical and basic neuroscientists, and patient-advocates, who met January 30-February 2, 2019, in Cannes (France) under the auspices of the European Association of Nuclear Medicine (EANM) to discuss the current role and the future potential of PET neuroimaging in dementia. The members of the EANM Neuroimaging

6 Department of Nuclear Medicine and Molecular Imaging, University of Groningen, University Medical Center Groningen, Groningen, The Netherlands

7 Department of Clinical Physiology, Nuclear Medicine and PET, Copenhagen University Hospital, Rigshospitalet, Copenhagen, Denmark

8 Nuclear Medicine Unit, IRCCS Ospedale Policlinico San Martino, Genoa, Italy

9 AMC Department of Radiology and Nuclear Medicine, Amsterdam University Medical Centers, Amsterdam, The Netherlands

10 Department of Nuclear Medicine, Medical Faculty and University Hospital Cologne, University of Cologne, Cologne, Germany

11 German Center for Neurodegenerative Diseases (DZNE), Bonn/ Köln, Germany

12 Institute of Neuroscience and Medicine, Molecular Organization of the Brain (INM-2), Forschungzentrum Jülich, Jülich, Germany 
Fig. 1 Examples of neuroimaging scans obtained with MRI or PET for primarily clinical use (left) to mainly research-oriented application (right). TSPO translocator protein, SV2A synaptic vesicle glycoprotein

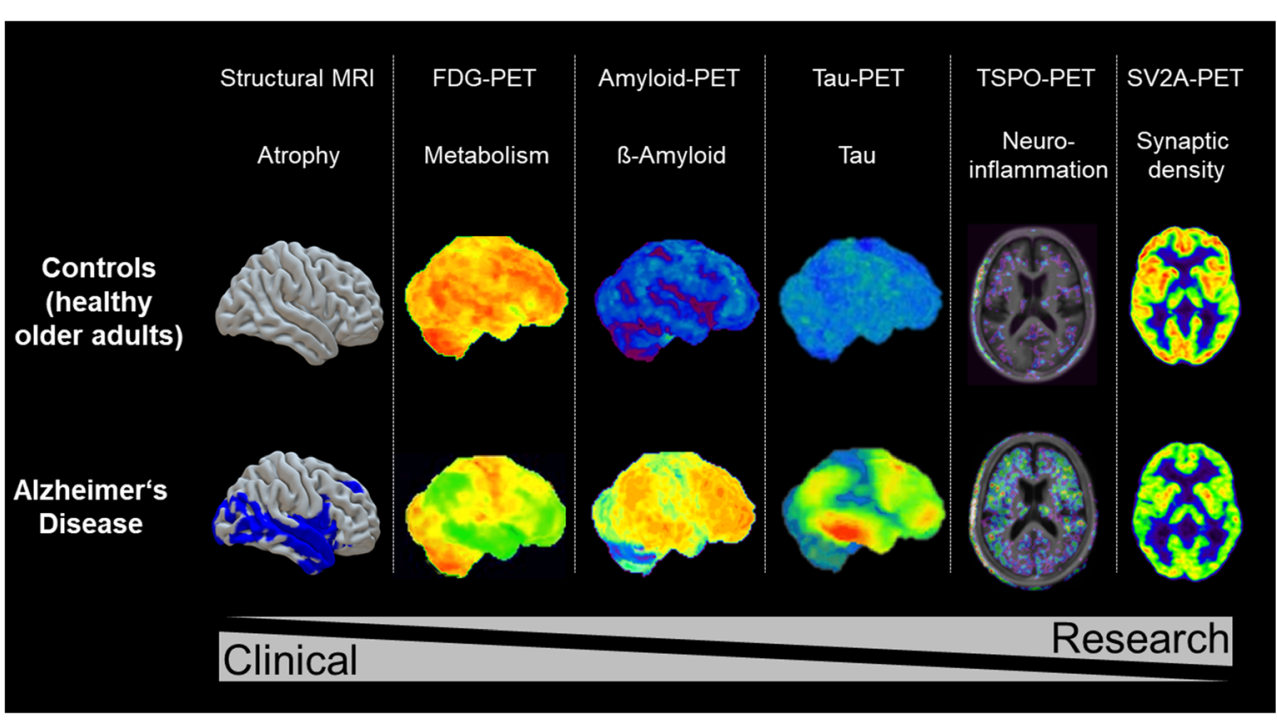

committee involved in this task by that time were invited to summarize the results of this paper for the European Journal of Nuclear Medicine and Molecular Imaging. To reach agreement, the specific strengths, respective and relative advantages, and disadvantages of each biomarker were carefully reviewed, weighted, and discussed in a structured process throughout five tracks, covering their use for early and differential diagnosis, clinical acceptance, use in research, and future developments, each ending with a controversy discussion followed by a consensus achievement.

We started by acknowledging the respective strengths and limitations of CSF versus PET biomarkers. This has particular relevance with regard to assessment of amyloid-pathology, where both CSF and imaging biomarkers are established and available. Briefly, CSF assessment is less expensive while amyloid-PET allows for better staging and monitoring of the extent and location of pathology. In addition, amyloid-PET can be applied when lumbar puncture is contraindicated or refused by the patient. The experts proposed to use these biomarkers also depending on local availability and expertise.

Next, the respective utility of the most validated imaging biomarkers for dementia were reviewed, which actually are already used in many academic memory clinics to support assessment and management of patients, and their specific utility in each of the main groups of neurodegenerative diseases (Alzheimer's disease, frontotemporal lobar degeneration (FTLD) spectrum, and parkinsonian syndromes (PS)).

What was agreed across panellists was that:

- Structural imaging should be proposed as a first step (following clinical and neuropsychological evaluation), as it allows for detection of other pathologies that could be responsible for cognitive decline, helps to assess presence and degree of co-morbidities, and because both topographical patterns and degree of atrophy are associated with specific neurodegenerative conditions and their severity. Yet, specificity is low and sensitivity is lower than, e.g., FDG-PET for neurodegeneration, so that additional biomarker assessment is often required;

- FDG-PET imaging has proved to be useful for early diagnosis since it can show the AD characteristic patterns of neurodegeneration at the mild cognitive impairment (MCI) stage earlier than MRI; it even has demonstrated added value to predict clinical outcome in people with MCI and a positive amyloid-PET, as a normal FDGPET scan would predict clinical stability while an abnormal FDG-PET would indicate cognitive deterioration, independently from the result of the amyloid test. Finally, it is useful for staging of disease and for differential diagnosis, because the patterns of brain hypometabolism are closely associated with type and severity of cognitive deficits, and they are distinct in different neurodegenerative diseases. Its main limitation, however, is that the detected patterns of hypometabolism do not allow to draw definite conclusions with regard to the underlying neuropathology.

- Amyloid-PET imaging on the other hand allows noninvasive in vivo detection of amyloid plaques - one of the main neuropathological landmarks of AD — with very high sensitivity and specificity. It is considered to have great value in reliably establishing the etiological diagnosis of $\mathrm{AD}$ in patients suffering from dementia, including clinically atypical variants of AD. A negative amyloidscan on the other hand can exclude AD as a reason for cognitive impairment. In large prospective trials, amyloid-imaging has demonstrated to result in changes 
in management in more than half of the patients studied. Amyloid-PET is considered to have higher sensitivity than FDG-PET for predicting progression from MCI to AD dementia, while FDG-PET has higher specificity and better accuracy for predicting short-term progression. According to appropriate use criteria [9], amyloidimaging is recommended in MCI, in patients with atypical clinical presentation, suspected mixed pathology or with early-onset cognitive decline. Panellists agreed that it could have utility beyond these indications and ongoing studies are, e.g., testing its value in subjects with subjective cognitive decline [10]. Amyloid-imaging does not allow for differentiation between distinct amyloidpositive disorders, and positive amyloid scans are found in about one third of cognitively unimpaired elderly with yet unknown clinical relevance [11].

As for the specific cases of FTLD spectrum and PS, which represent frequent differential diagnoses to $\mathrm{AD}$, the panellists agreed that:
- In the FTLD spectrum, FDG-PET has a long-standing role based on signature patterns of hypometabolism associated with specific clinical syndromes, while amyloidPET has proven useful in discriminating AD from FTLD, as amyloid plaques are typically not part of the FTLD neuropathological spectrum. Amyloid-PET is thus useful when a differential diagnosis between $\mathrm{AD}$ and non-AD causes of dementia is needed, while FDG-PET can further address differential diagnosis within the FTLD spectrum in patients who are amyloid-negative, or when the final diagnosis is still unclear after amyloid-PET or CSF analyses.

- As for PS, assessment of integrity of the nigrostriatal dopaminergic pathway with presynaptic dopaminergic imaging, such as DaT-SPECT or FDOPA-PET, is recommended and approved by regulatory authorities to support the differential diagnosis between PS and AD. It may not be sufficient, however, as dopaminergic deficits can be present in all neurodegenerative PS. Therefore, FDG-PET can be very useful here to distinguish between the different PS.

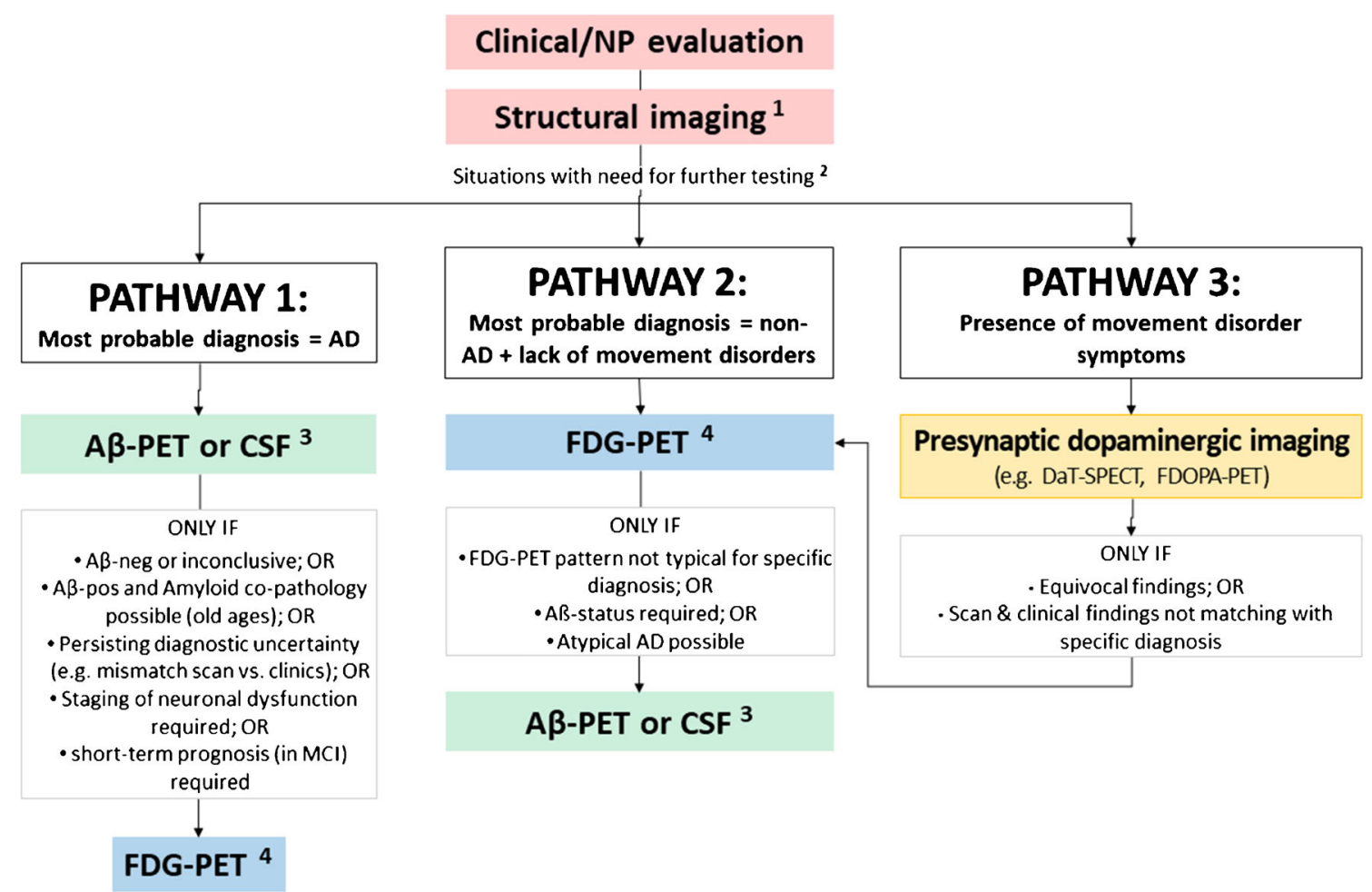

Fig. 2 Proposed algorithm for early and differential diagnoses of dementia. ${ }^{1}$ Exclusionary and inclusionary role: exclusion of neoplastic, vascular, and inflammatory changes supporting non-neurodegenerative aetiologies and evaluation of topography of atrophy might inform on the neurodegenerative disease (but FDG-PET might be more sensitive and accurate). ${ }^{2}$ Additional biomarker assessment is required after clinical and structural imaging information if tailored therapy concepts are the aim or if decisions depend on a conclusive diagnosis and prognosis. In contrast, if clinical and structural imaging information both converge towards a specific diagnosis, or if consequences of the diagnosis are limited (e.g., other comorbidities dominating patient prognosis), then no further assessment is needed. ${ }^{3}$ Whatever is established/available and preferred; always A $\beta$-PET if CSF is contraindicated or inconclusive. ${ }^{4}$ Age and APOE status (when available) may influence the use of FDGPET even before amyloid-PET especially in individuals with available but inconclusive CSF results. Analyses of FDG-PET images should also take into account comorbidities, i.e., uncontrollable diabetes, brain trauma, chronic ischemia, and some medications (e.g., psychotropic drugs or corticosteroids) that can alter cerebral metabolism 
Based on these different statements, the expert panel proposed an algorithm with 3 possible pathways with distinct sequences of biomarkers, depending on the clinical presentation of the patients (Fig. 2). As mentioned above, the 3 pathways start with clinical and neuropsychological examinations followed by structural MRI, whenever possible. In cases where AD appears to be the most probable diagnosis, an amyloid-PET scan is recommended next (if there are uncertainties or inconsistencies from previous examinations or if information on amyloid status is required), followed by an FDG-PET scan in specific situations that require further information, e.g., on short-term prognosis. The second pathway is recommended when $\mathrm{AD}$ does not appear to represent the most probable diagnosis and no characteristic symptoms for a movement disorder are present. The panel of experts argued that in these cases, the amyloid status is more likely to be negative, which would potentially not contribute much to the differential diagnosis, while FDG-PET could provide conclusive information on the specific (amyloid-negative) degenerative diseases. From there, an amyloid-PET scan could be proposed only in cases who do not show an FDG-PET pattern typical for a specific diagnosis, if atypical $\mathrm{AD}$ is possible or if the amyloid status is required. Finally, the third pathway concerns patients presenting with movement disorder symptoms in which the first step would obviously be to confirm the presence of dopaminergic deficits indicative of neurodegenerative PS with presynaptic dopaminergic imaging. Next, FDG-PET would be proposed to further specify the diagnosis amongst the different neurodegenerative PS, or if diagnostic uncertainties remain. Finally, amyloid testing might be necessary in few cases where atypical AD remains possible.

It is both early and late to propose this diagnostic algorithm. Early because no standard answers can be provided yet with regard to cost efficicacy and efforts needed to generalize the suggested practice and because prospective data on clinical utility are still being collected on some of the biomarkers; late because biomarkers are already in use, but inconsistently, e.g., in many academic memory clinics, so that there is obvious need for consensual recommendations. Because the goal was to define priority and useful order of the best validated biomarkers already in clinical use, we did not include rapidly developing and upcoming biomarkers such as tau-PET or blood biomarkers. This would need to be incorporated in the future as (i) blood biomarkers are rapidly evolving and demonstrating efficiency and (ii) one tau-PET tracer (flortaucipir F18; Tauvid; Avid Radiopharmaceuticals) already received approval from the US Food and Drug Administration for patients being evaluated for AD. Similarly, dual-phase imaging and integrated PET/MRI are other on-going developments that have potential to impact the proposed algorithm in the future, allowing the acquisition of multiple biomarkers in a one-stop manner.

\section{Declarations}

Ethical approval Institutional Review Board approval was not required because the paper is an Editorial.

Informed consent Not applicable.

Conflict of interest GC has received research support from the EU's Horizon 2020 research and innovation programme (grant agreement number 667696), Inserm, Fondation d'entreprise MMA des Entrepreneurs du Futur, Fondation Alzheimer, Programme Hospitalier de Recherche Clinique, Région Normandie, Association France Alzheimer et maladies apparentées and Fondation Vaincre Alzheimer (all to Inserm), and personal fees from Fondation d'entreprise MMA des Entrepreneurs du Futur.

AD reports personal fees and non-financial support from General Electric Healthcare, Life Molecular Imaging (Piramal), Lilly, during the conduct of the study, grants, and personal fees and non-financial support from Siemens Healthcare, outside of the submitted work, and has a PSMA patent pending.

JA reports grants from General Electric, Piramal, and Lilly-Avid, personal fees from Biogen and Bayer, personal fees and non-financial support from Araclon and Advanced Accelerator Applications, outside of the submitted work.

VG is supported by the Swiss National Science Foundation (projects 320030_169876 and 320030_185028), the Velux foundation (project 1123), the Aetas foundation, and the Association Pour la Recherche sur l'Alzheimer; reports grants from Siemens Healthineers, Life Molecular Imaging, Merck, Cerveau Technologies, Roche, and General Electric Healthcare; and personal fees from Siemens Healthineers and General Electric Healthcare, outside the submitted work.

The other authors declare no conflicts of interest.

\section{References}

1. McKhann G, Drachman D, Folstein M, Katzman R, Price D, Stadlan EM. Clinical diagnosis of Alzheimer's disease: report of the NINCDS-ADRDA Work Group under the auspices of Department of Health and Human Services Task Force on Alzheimer's Disease. Neurology. 1984;34:939-44.

2. Jack CR, Bennett DA, Blennow K, Carrillo MC, Dunn B, Haeberlein SB, et al. NIA-AA research framework: toward a biological definition of Alzheimer's disease. Alzheimers Dement. 2018;14:535-62. https://doi.org/10.1016/j.jalz.2018.02.018.

3. McKhann GM, Knopman DS, Chertkow H, Hyman BT, Jack CR $\mathrm{Jr}$, Kawas CH, et al. The diagnosis of dementia due to Alzheimer's disease: recommendations from the National Institute on AgingAlzheimer's association workgroups on diagnostic guidelines for Alzheimer's disease. Alzheimers Dement. 2011;7:263-9. https:// doi.org/10.1016/j.jalz.2011.03.005.

4. Dubois B, Feldman HH, Jacova C, Hampel H, Molinuevo JL, Blennow K, et al. Advancing research diagnostic criteria for Alzheimer's disease: the IWG-2 criteria. Lancet Neurol. 2014;13: 614-29. https://doi.org/10.1016/S1474-4422(14)70090-0.

5. Garrett MD. A critique of the 2018 National Institute on Aging's. Research framework: toward a biological definition of Alzheimer's disease. Curr Neurobiol. 2018;9:49-58.

6. Laforce R, Soucy J-P, Sellami L, Dallaire-Théroux C, Brunet F, Bergeron D, et al. Molecular imaging in dementia: past, present, 
and future. Alzheimers Dement. 2018;14:1522-52. https://doi.org/ 10.1016/j.jalz.2018.06.2855.

7. Frisoni GB, Boccardi M, Barkhof F, Blennow K, Cappa S, Chiotis $\mathrm{K}$, et al. Strategic roadmap for an early diagnosis of Alzheimer's disease based on biomarkers. Lancet Neurol. 2017;16:661-76. https://doi.org/10.1016/S1474-4422(17)30159-X.

8. Chételat G, Arbizu J, Barthel H, Garibotto V, Law I, Morbelli S, et al. Amyloid-PET and 18F-FDG-PET in the diagnostic investigation of Alzheimer's disease and other dementias. Lancet Neurol. 2020;19:951-62. https://doi.org/10.1016/S1474-4422(20)30314-8.

9. Johnson KA, Minoshima S, Bohnen NI, Donohoe KJ, Foster NL, Herscovitch P, et al. Appropriate use criteria for amyloid PET: a report of the Amyloid Imaging Task force, the Society of Nuclear Medicine and Molecular Imaging, and the Alzheimer's
Association. Alzheimers Dement. 2013;9:E1-16. https://doi.org/ 10.1016/j.jalz.2013.01.002.

10. Frisoni GB, Barkhof F, Altomare D, Berkhof J, Boccardi M, Canzoneri E, et al. AMYPAD diagnostic and patient management study: rationale and design. Alzheimers Dement. 2018. https://doi. org/10.1016/j.jalz.2018.09.003.

11. Jansen WJ, Ossenkoppele R, Knol DL, Tijms BM, Scheltens P, Verhey FRJ, et al. Prevalence of cerebral amyloid pathology in persons without dementia: a meta-analysis. JAMA. 2015;313: 1924-38. https://doi.org/10.1001/jama.2015.4668.

Publisher's note Springer Nature remains neutral with regard to jurisdictional claims in published maps and institutional affiliations. 